\title{
Chiral condensate and chemical freeze-out
}

\author{
D. B. Blaschke* \\ Institute for Theoretical Physics, University of Wroclaw, 50-204 Wroclaw, Poland and \\ Bogoliubov Laboratory of Theoretical Physics, JINR, 141980 Dubna, Russia \\ J. Berdermann \\ DESY Zeuthen, D-15738 Zeuthen, Germany \\ J. Cleymans \\ UCT-CERN Research Centre and Department of Physics, \\ Rondebosch 7r01, Cape Town, South Africa \\ K. Redlich \\ Institute for Theoretical Physics, University of Wroclaw, 50-204 Wroclaw, Poland and \\ ExtreMe Matter Institute EMMI, GSI, D-64291 Darmstadt, Germany
}

(Dated: February 16, 2011)

\begin{abstract}
We consider a chemical freeze-out mechanism which is based on a strong medium dependence of the rates for inelastic flavor-equilibrating collisions based on the delocalization of hadronic wave functions and growing hadronic radii when approaching the chiral restoration. We investigate the role of mesonic (pion) and baryonic (nucleon) fluctuations for melting the chiral condensate in the phase diagram in the $(T, \mu)$-plane. We apply the PNJL model beyond mean-field and present an effective generalization of the chiral perturbation theory result which accounts for the medium dependence of the pion decay constant while preserving the GMOR relation. We demonstrate within a schematic resonance gas model consisting of a variable number of pionic and nucleonic degrees of freedom that within the above model a quantitative explanation of the hadonic freeze-out curve and its phenomenological conditions can be given.
\end{abstract}

PACS numbers: 12.38.Mh,25.75.-q,24.10.Pa

*Electronic address: blaschke@ift.uni.wroc.pl 


\section{INTRODUCTION}

The investigation of the phase diagram of QCD in the plane of temperature $T$ and baryochemical potential $\mu_{B}$ is one of the goals of heavy-ion collision experiments, of lattice QCD (LQCD) and effective field theory approaches to the nonperturbative sector of QCD. Of particular interest are the conditions under which the approximate chiral symmetry of the QCD Lagrangian will get restored and whether this transition will be necessarily accompanied by the deconfinement of quarks and gluons. More detailed questions to the QCD phase diagram concern the order of these phase transitions, their critical exponents and fluctuation measures as well as the possible existence of a critical point or even a triple point in the phase diagram. Most promising tools for the experimental determination of these characteristics are the energy scan programs at CERN, RHIC and at the upcoming dedicated facilities of the third generation: FAIR and NICA. The systematic analysis of higher moments of distributions of produced particles in their dependence on the collision energy and the size of collising systems shall provide answers to the above questions and allow direct comparison with predictions from the underlying theory, as provided by LQCD, see, e.g., Ref. [1] and works cited therein.

As long as the applicability of LQCD methods is bound to the region of finite $T$ and $\mu_{B} / T \ll 1$, any predictions for the phase structure of QCD at high baryon densities including the possible existence of critical points will rely on effective models. To be relevant for the discussion of the above problems these models have to share with QCD the property of chiral symmetry and its dynamical breaking as well as a mechanism for confinement and deconfinement.

At present stage, of particular relevance for the discussion of the QCD phase diagram are the chemical freeze-out parameters $\left(T^{f}, \mu_{B}^{f}\right)$ which have been obtained from the statistical model analysis of particle yields obtained in heavy ion collisions [2, 3]. One of the most striking observations is the systematic behaviour of these parameters with collision energy $\sqrt{s}$ [4 6 ], which has recently been given a simple parametric form [7]. It has been observed that the resulting freeze-out curve in the phase diagram is closely correlated to the thermodynamical quantities of the hadron resonance gas described by the statistical model. These phenomenological freeze-out conditions make statements about the mean energy per hadron $\langle E\rangle /\langle N\rangle \simeq 1.0 \mathrm{GeV}$, the dimensionless entropy density $s / T^{3} \simeq 7$ and a total baryon and 
antibaryon density $n_{B}+n_{\bar{B}} \simeq 0.12 \mathrm{fm}^{-3}$. The freeze-out line provides a lower bound for the chiral restoration and deconfinement transition in the phase diagram. Being coincident at low densities as inferred from LQCD [8], both transitions need not to occur simultaneously at high density thus allowing for an island of a quarkyonic phase [9] between hadron gas and quark-gluon plasma with a (pseudo-)triple point [10].

The question appears for the physical mechanism which governs the chemical freeze-out and which determines quantitatively the freeze-out parameters. One aspect is provided by the requirement that hadrons should overlap in order to facilitate flavor exchange reactions which establish chemical equilibrium. This geometrical picture of freeze-out is sucessfully realized in a percolation theory approach [11]. Another aspect is the dynamical one: when the equation of state (EoS) possess softest points (e.g., due to the dissociation of hadrons into their quark and gluon constituents with a sufficient release of binding energy involved) which is correlated with the freeze-out curve in the phase diagram, then it is obvious that the hadron abundances are characterized by the corresponding $T$ and $\mu_{B}$ values [12]. The dynamical system got quasi trapped at the softest points for sufficient time to achieve chemical equilibration before evaporating as a gas of hadron resonances freely streaming to the particle detectors. Also the kinetic aspect of fast chemical equilibration was discussed in the hadronic gas when accounting for a multi-hadron dynamics [13].

All these mechanisms are appealing since they provide an intuitively clear picture, but they are flawed by the fact that their relation to fundamental aspects of the QCD phase transition like the chiral condensate as an order parameter are not an element of the description.

In the present contribution we develop an approach which relates the geometrical as well as the hydrodynamic and kinetic aspects of chemical freeze-out to the medium dependence of the chiral condensate. We demonstrate within a beyond-meanfield extension [14 20] of the Polyakov NJL model [21 27] how the excitation of hadronic resonances initiates the melting of the chiral condensate which entails a Mott-Anderson type delocalization of the hadron wave functions and a sudden drop in the relaxation time for flavor equilibration. Already for a schematic resonance gas consiting of pions and nucleons with artificially enhaced numbers of degrees of freedom we can demonstrate that a kinetic freeze-out condition for the above model provides quantitative agreement with the phenomenological freeze-out curve. 


\section{CHEMICAL FREEZE-OUT AND CHIRAL CONDENSATE}

We propose to relate the chemical freeze-out to the chiral condensate in the following effective way. As a freeze-out condition for flavor equilibrating reaction kinetics in the temperature-chemical potential plane $(T, \mu)$ we employ

$$
\tau_{\exp }(T, \mu)=\tau_{\text {coll }}(T, \mu)
$$

where $\tau_{\exp }(T, \mu)$ is the expansion time scale of the hadronic fireball and the inverse of the relaxation time for reactive collisions is

$$
\tau_{\text {coll }}^{-1}(T, \mu)=\sum_{i, j} \sigma_{i j} n_{j}
$$

with $i, j=\pi, N, \ldots$ running over all species in the hadron resonance gas. For the cross sections we adopt the geometrical Povh-Hüfner law [28, 29]

$$
\sigma_{i j}=\lambda\left\langle r_{i}^{2}\right\rangle\left\langle r_{j}^{2}\right\rangle
$$

where $\lambda$ is a constant of the order of the string tension $\lambda \sim 1 \mathrm{GeV} / \mathrm{fm}=5 \mathrm{fm}^{-2}$. Note that this behaviour has been obtained for the quark exchange contribution to hadron-hadron cross sections [30].

A key point of our approach is that the radii of hadrons shall depend on $T$ and $\mu$ and shall diverge when hadron dissociation (Mott effect) sets in, driven basically by the restoration of

chiral symmetry. This has quantitatively been studied for the pion [31], where it has been shown that close to the Mott transition the chiral perturbation theory corrections can be safely neglected and the pion radius is well approximated by

$$
r_{\pi}^{2}(T, \mu)=\frac{3}{4 \pi^{2}} f_{\pi}^{-2}(T, \mu)
$$

It has been demonstrated that the GMOR relation holds out to the chiral phase transition where pions would merge the continuum of unbound quark matter [32, 33]. Since the current quark mass is $T$ - and $\mu$-independent and the pion mass is "chirally protected", the $T-$, $\mu$-dependence of the chiral condensate has to be reflected in a similar behaviour of the pion decay constant

$$
f_{\pi}^{2}(T, \mu)=-m_{0}\langle\bar{q} q\rangle_{T, \mu} / M_{\pi}^{2}
$$


The resulting relationship between pion radius and chiral condensate in the medium reads

$$
r_{\pi}^{2}(T, \mu)=\frac{3 M_{\pi}^{2}}{4 \pi^{2} m_{q}}\left|\langle\bar{q} q\rangle_{T, \mu}\right|^{-1} .
$$

The delocalization of the pion wave function due to the melting of the chiral condensate as expressed in this formula is the most important element of the hadronic freeze-out mechanism suggested in this work.

For the nucleon, we shall assume the radius to consist of two components, a medium independent hard core radius $r_{0}$ and a pion cloud contribution

$$
r_{N}^{2}(T, \mu)=r_{0}^{2}+r_{\pi}^{2}(T, \mu),
$$

where from the vacuum values $r_{\pi}=0.59 \mathrm{fm}$ and $r_{N}=0.74 \mathrm{fm}$ one gets $r_{0}=0.45 \mathrm{fm}$.

For the expansion time scale we adopt a relationship which follows from entropy conservation, $S=s(T, \mu) V\left(\tau_{\exp }\right)=$ const, and a fireball expansion law $V\left(\tau_{\exp }\right)$. Assuming that $V\left(\tau_{\exp }\right) \propto \tau_{\exp }^{3}$ one obtains

$$
\tau_{\exp }(T, \mu)=a s^{-1 / 3}(T, \mu),
$$

with $a$ being a constant of the order one.

As a first step, we will restrict the discussion to a medium consisting of pions and nucleons only, whereby we apply the above relationships. The generalization to a hadron resonance gas along these lines is rather straightforward.

In the following we introduce properties of the chiral condensate in a pion-nucleon and in a hadron resonance gas and then applying the freeze-out condition (2) we compare our model results with phenomenological findings on hadronic freeze-out and its conditions.

\section{CHIRAL CONDENSATE BEYOND MEANFIELD}

We start from the general definition of the chiral condensate

$$
\langle\bar{q} q\rangle=\frac{\partial}{\partial m_{0}} \Omega(T, \mu) .
$$

The thermodynamical potential $\Omega(T, \mu)$ can be decomposed into contributions from the quark and gluon meanfield and hadronic (quantized) fluctuations in the meson and baryon channels

$$
\Omega(T, \mu)=\Omega_{\mathrm{MF}}(T, \mu)+\Omega_{\text {meson }}(T, \mu)+\Omega_{\text {baryon }}(T, \mu)-\Omega(0,0) .
$$


The subtraction of $\Omega(0,0)$ removes vacuum divergencies and guarantees that the thermodynamical potential, i.e., pressure and energy density, of the vacuum vanish.

The mean field contribution is given by [36]

$$
\begin{aligned}
\Omega_{\mathrm{MF}}(T, \mu) & =-2 N_{f} \int \frac{d^{3} p}{(2 \pi)^{3}}\left\{3 \varepsilon_{p}+T \ln \left[1+3 \Phi e^{-\beta\left(\varepsilon_{p}-\mu\right)}+3 \Phi e^{-2 \beta\left(\varepsilon_{p}-\mu\right)}+e^{-3 \beta\left(\varepsilon_{p}-\mu\right)}\right]\right. \\
& \left.+T \ln \left[1+3 \Phi e^{-\beta\left(\varepsilon_{p}+\mu\right)}+3 \Phi e^{-2 \beta\left(\varepsilon_{p}+\mu\right)}+e^{-3 \beta\left(\varepsilon_{p}+\mu\right)}\right]\right\}+\frac{\sigma^{2}}{4 G}+\mathcal{U}(\Phi ; T),(11)
\end{aligned}
$$

where the quasiparticle energy is $\varepsilon_{p}=\sqrt{p^{2}+m^{2}(T, \mu)}$, and $m(T, \mu)=m_{0}+\sigma(T, \mu)$. In the following we will shorten the notation by dropping the arguments of $m(T, \mu)=m$ and $\sigma(T, \mu)=\sigma$. For the Polyakov-loop potential we take the logarithmic form motivated by the $\mathrm{SU}(3)$ Haar measure,

$$
\mathcal{U}(\Phi, T)=\left[-\frac{1}{2} a(T) \Phi^{2}+b(T) \ln \left(1-6 \Phi^{2}+8 \Phi^{3}-3 \Phi^{4}\right)\right] T^{4},
$$

with the corresponding definitions of $a(T)$ and $b(T)$ [16]. In Ref. [14], a dependence of the $T_{0}$ parameter on the number of active flavors and on the chemical potential has been suggested. Here we will use $T_{0}=200 \mathrm{MeV}$. The mean field value of the traced Polyakov loop is then given by $\bar{\Phi}=\bar{\Phi}^{*}=\left[1+2 \cos \left(\bar{\phi}_{3} / T\right)\right] / 3$.

The quark mass gap is obtained from the extremum condition $\partial \Omega / \partial \sigma=0$, equivalent to

$$
\frac{\sigma}{2 G}=\frac{6}{\pi^{2}} \int d p p^{2} \frac{m}{\varepsilon_{p}}\left[1-f_{\Phi}^{+}-f_{\Phi}^{-}\right],
$$

where the PNJL quark distribution functions are given by

$$
f_{\Phi}^{ \pm}=\frac{\Phi\left[e^{-\beta\left(\varepsilon_{p}-\mu\right)}+2 e^{-2 \beta\left(\varepsilon_{p}-\mu\right)}\right]+e^{-3 \beta\left(\varepsilon_{p}-\mu\right)}}{1+3 \Phi e^{-\beta\left(\varepsilon_{p}-\mu\right)}+3 \Phi e^{-2 \beta\left(\varepsilon_{p}-\mu\right)}+e^{-3 \beta\left(\varepsilon_{p}-\mu\right)}} .
$$

The values of the Polyakov loop $\Phi$ in the phase diagram are found from a similar gap equation corresponding to the solution of the extremum condition $\partial \Omega / \partial \Phi=0$.

The contribution of mesonic fluctuations has been discussed in [34, 35] for the NJL model and in [36, 37] for the PNJL model. Also recently mesonic fluctuations were included in these models within the functional renormalization group approach [38, 39].

In the following we consider only the on-mass-shell meson contributions and neglect the continuum correlations beyond the Mott effect. This is justified because we are actually interested in the hadronic freeze-out occurring in a region of the phase diagram which does not exceed the limits of the hadronic phase. This contribution is given as

$$
\Omega_{\text {meson }}(T, \mu)=\sum_{M=\pi, \ldots} d_{M} \int \frac{d^{3} k}{(2 \pi)^{3}}\left\{\frac{E_{M}(k)}{2}+T \ln \left[1-e^{-\beta E_{M}(k)}\right]\right\},
$$


where the index $M$ denotes the actual meson with degeneracy factor $d_{M}$. We restrict this sum to the lowest meson state, the pion, with $E_{\pi}(k)=\sqrt{k^{2}+M_{\pi}^{2}}$.

The contribution of pionic fluctuations to the chiral condensate we obtain from

$$
\frac{\partial \Omega_{\pi}}{\partial m_{0}}=\langle\bar{q} q\rangle_{\pi}=-\frac{M_{\pi} n_{s, \pi}}{2 m_{0}}+\langle\bar{q} q\rangle_{\pi}^{\mathrm{vac}}
$$

where the GMOR relation has been used to evaluate $\partial E_{\pi}(k) / \partial m_{0}=M_{\pi}^{2} /\left(2 m_{0} E_{\pi}(k)\right)$ and the scalar pion density has been defined as

$$
n_{s, \pi}=\frac{d_{\pi}}{2 \pi^{2}} \int_{0}^{\infty} d p p^{2} \frac{M_{\pi}}{E_{\pi}(p)} \frac{1}{e^{\beta E_{\pi}(p)}-1}
$$

The last term corresponds to a vacuum contribution which gets eliminated by the vacuum subtraction rule (10) inherent in the definition of the full condensate (9).

The contribution of baryons to the partition function is considered as an ideal Fermi gas

$$
\Omega_{\text {baryon }}(T, \mu)=-\sum_{B=N, \ldots} d_{B} \int \frac{d^{3} k}{(2 \pi)^{3}}\left\{\frac{E_{B}(k)}{2}+T \ln \left[1+e^{-\beta\left(E_{B}(k)-\mu_{B}\right)}\right]\right\}+\mu_{B} \leftrightarrow-\mu_{B}(18)
$$

For the discussion of the quark condensate we will focus here on the nucleonic contribution

$$
\frac{\partial \Omega_{N}}{\partial m_{0}}=\langle\bar{q} q\rangle_{N}=-\frac{\sigma_{N} n_{s, N}(T, \mu)}{m_{0}}+\langle\bar{q} q\rangle_{N}^{\mathrm{vac}}
$$

where we have used the Feynman-Hellman theorem and introduced the pion-nucleon sigma term, $\sigma_{N}=m_{0}\left(\partial m_{N} / \partial m_{0}\right)=45 \mathrm{MeV}$ [40]. The nucleon scalar density is given by

$$
n_{s, N}(T, \mu)=\frac{d_{N}}{2 \pi^{2}} \int_{0}^{\infty} d p p^{2} \frac{m_{N}}{E_{N}(p)}\left\{f_{N}(T, \mu)+f_{N}(T,-\mu)\right\}
$$

where $f_{N}(T, \mu)=\left\{1+\exp \left[\left(\sqrt{p^{2}+m_{N}^{2}}-\mu_{B}\right) / T\right]\right\}^{-1}$ is the nucleon Fermi distribution, $m_{N}=939 \mathrm{MeV}$ is the nucleon mass and $\mu_{B}=3 \mu$ the baryon chemical potential. The vacuum contribution stemming from the zero-point energy term gets removed by the vacuum subtraction procedure (10).

From the above discussion, the chiral condensate in a $\pi-N$ gas can be obtained as

$$
-\langle\bar{q} q\rangle=\frac{\sigma}{2 G}-\frac{M_{\pi} n_{s, \pi}(T)}{2 m_{0}}-\frac{\sigma_{N} n_{s, N}(T, \mu)}{m_{0}} .
$$

Note, that using the chiral limit expression for the scalar pion density $n_{s, \pi}=d_{\pi} M_{\pi} T^{2} / 12$, and the GMOR relation (5), we may give this result the form

$$
\langle\bar{q} q\rangle=\langle\bar{q} q\rangle_{\mathrm{MF}}\left[1-\frac{T^{2}}{8 f_{\pi}^{2}(T, \mu)}-\frac{\sigma_{N} n_{s, N}(T, \mu)}{M_{\pi}^{2} f_{\pi}^{2}(T, \mu)}\right]
$$


which is known from the chiral perturbation theory [41, 42], but now with a mediumdependent pion decay constant. Since the modification of the meanfield contribution stemming from the quark excitations is proportional to $f_{\Phi}$, it is now effectively suppressed by the Polyakov-loop, compared to the standard NJL model case.

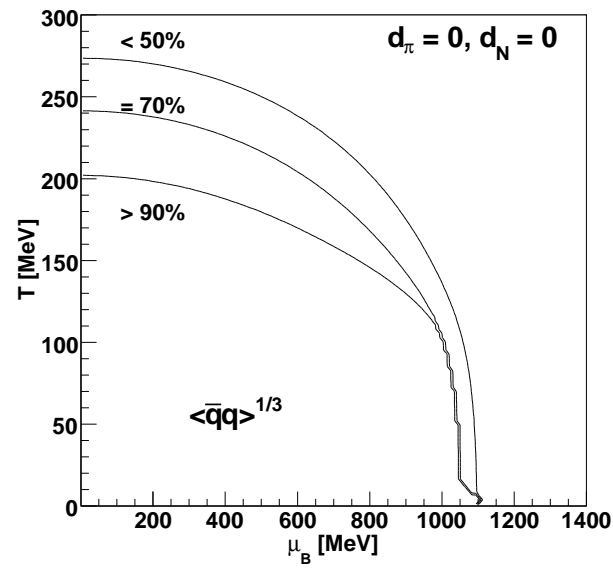

(a)

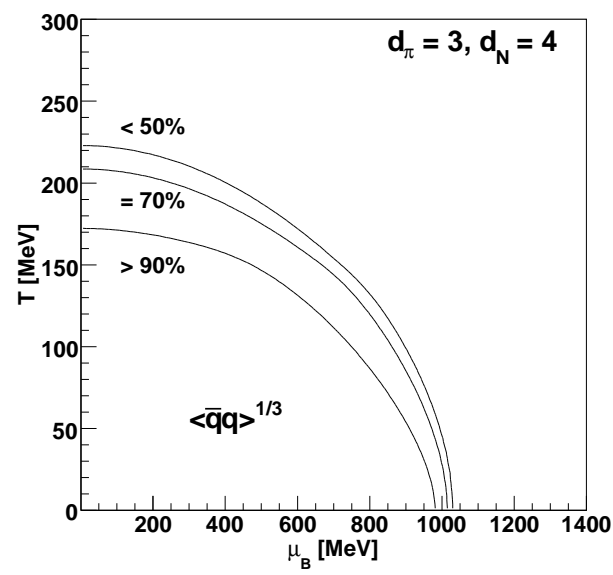

(c)

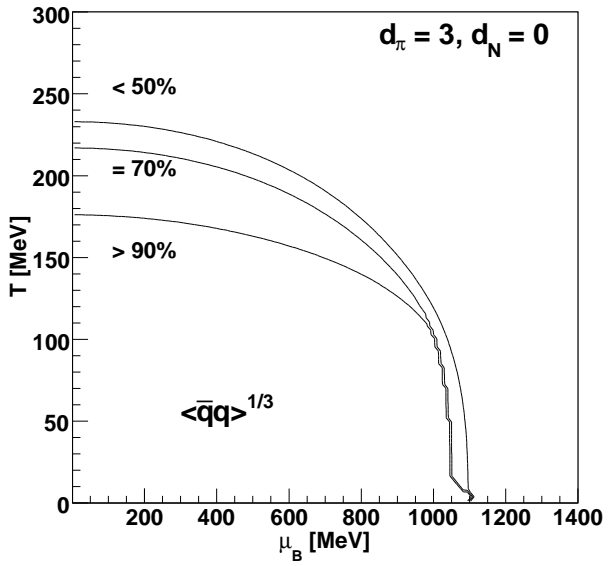

(b)

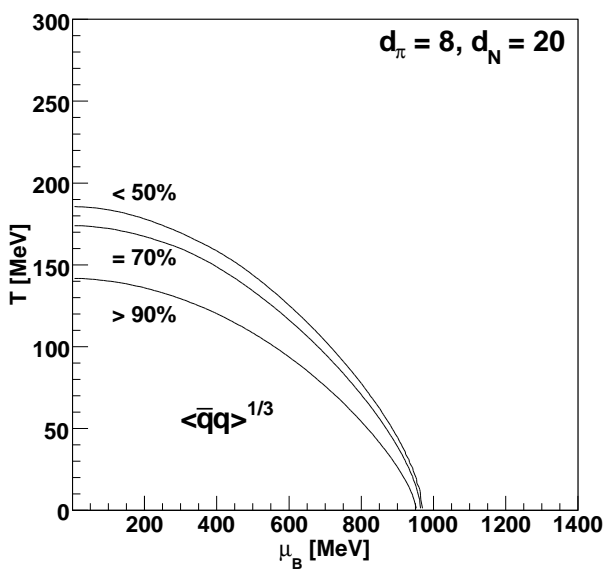

(d)

FIG. 1: Behavior of the chiral condensate $\langle q \bar{q}\rangle$ in the PNJL model at meanfield level (a), with pion fluctuations (b), with pion and nucleon fluctuations (c) and for the schematic resonance gas medium with 8 pionic and 20 nucleonic degrees of freedom (d). Also shown are the corresponding reductions of the chiral condensate relative to its vacuum value for each set of parameters.

Numerical results for the condensate in the $T-\mu$ plane are given in Fig. 1, where we display contours of equal condensate and indicate its percentage reduction relative to the vacuum value. We use standard values of the NJL model parametrization, e.g., from [43], 
with $G \Lambda^{2}=2.31825, \Lambda=602.472 \mathrm{MeV}, m_{0}=5.27697 \mathrm{MeV}, m_{s, 0}=150 \mathrm{MeV}, M_{\pi}=140$ $\mathrm{MeV}, M_{K}=495 \mathrm{MeV}, f_{\pi}=92.4 \mathrm{MeV}$ and $f_{K}=93.6 \mathrm{MeV}$.

\section{THE FREEZE-OUT CURVE}

In the following we compare predictions of our model on the freeze-out conditions with that obtained within the statistical model analysis of particle yields in heavy ion collisions.

Fig. 2 shows results for different freeze-out curves obtained from the kinetic condition, Eq. (11) applied to the schematic resonance gas model with variable numbers of pion $\left(d_{\pi}\right)$ and nucleon $\left(d_{N}\right)$ degrees of freedom. The results are compared with the phenomenological values. From this figure, it is clear that for $d_{\pi}=8$ and $d_{N}=14 \ldots 20$ there is an excellent

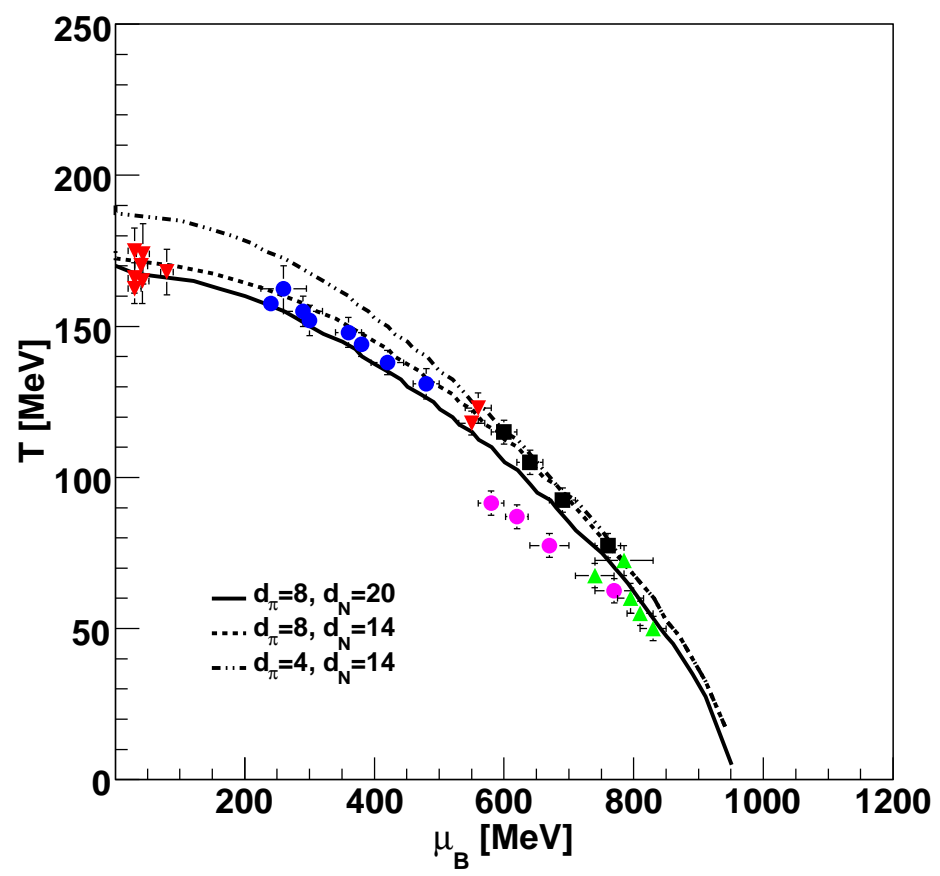

FIG. 2: Freeze-out curves according to the kinetic freeze-out condition, Eq. (1), for the schematic resonance gas model with different number of pion $\left(d_{\pi}\right)$ and nucleon $\left(d_{N}\right)$ degrees of freedom compared to phenomenological values (symbols) [10]. The lines correspond to different choices for $d_{\pi}$ and $d_{N}$ given in the legend.

correspondence of our freeze-out criterion with phenomenological values. Increasing $d_{\pi}$ results in decreasing freeze-out temperatures in the meson-dominated region at small $\mu_{B} / T$. 
On the other hand, increasing $d_{N}$ shifts the entire freeze-out curve towards lower $T$ and $\mu_{B}$. The freeze-out curves calculated within our model correspond to a reduction of the chiral condensate up to $70 \%$ of its vacuum value, c.f. Fig. 1(d), so that the hadron-hadron cross section in (2) according to (3) is roughly twice the vacuum value.

To verify further our model, we evaluate the entropy density $s(T, \mu)=-\partial \Omega / \partial T$, which according to the statistical model is related to the freeze-out curve by the phenomenological condition, $s\left(T^{f}, \mu_{B}^{f}\right) / T^{3} \simeq 7$. Fig. 3 (left) shows the comparison of the phenomenological freeze-out points with the lines of constant $s(T, \mu) / T^{3}$ calculated within our model. The dashed line in this figure corresponds to $s(T, \mu) / T^{3}=7$, which is the freeze-out line obtained within the hadron resonance gas model.

Fig. 3 (right) shows a similar comparison as the left-hand figure but assuming freeze-out conditions of constant density of baryons, $n_{B}\left(T^{f}, \mu_{B}^{f}\right)+\bar{n}_{B}\left(T^{f}, \mu_{B}^{f}\right)=$ const.. The nucleon density is obtained from

$$
n_{N}(T, \mu)=\frac{d_{N}}{2 \pi^{2}} \int_{0}^{\infty} k^{2} \mathrm{~d} k \frac{1}{1+e^{\beta\left(E_{N}(k)-\mu_{B}\right)}}
$$

where $d_{N}=20$ is the number of nucleonic degrees of freedom in our schematic resonance gas and the antinucleon density, $\bar{n}_{N}(T, \mu)=n_{N}(T,-\mu)$. The result of the calculation with the above parameters is shown in the right panel of Fig. 3. From this figure one can conclude that our effective model reproduces the phenomenological freeze-out line also when imposing conditions of fixed total density of baryons.

\section{CONCLUSION AND OUTLOOK}

We have developed chemical freeze-out mechanism which is based on a strong medium dependence of the rates for inelastic flavor-equilibrating collisions based on the delocalization of hadronic wave functions and growing hadronic radii when approaching the chiral restoration. This approach relates the geometrical (percolation) as well as the hydrodynamic (softest point) and kinetic (quark exchange) aspects of chemical freeze-out to the medium dependence of the chiral condensate. For our model calculations we have employed a beyond-mean-field effective extension of the Polyakov NJL model. We could demonstrate how the excitation of hadronic resonances initiates the melting of the chiral condensate which entails a Mott-Anderson type delocalization of the hadron wavefunctions and a sudden drop 


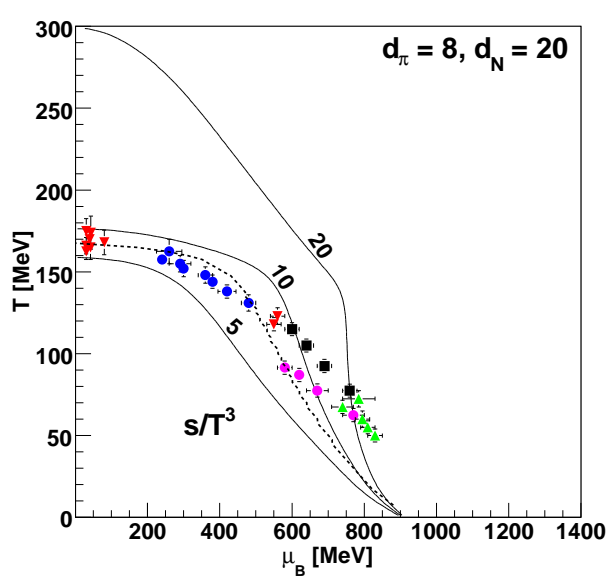

(a)

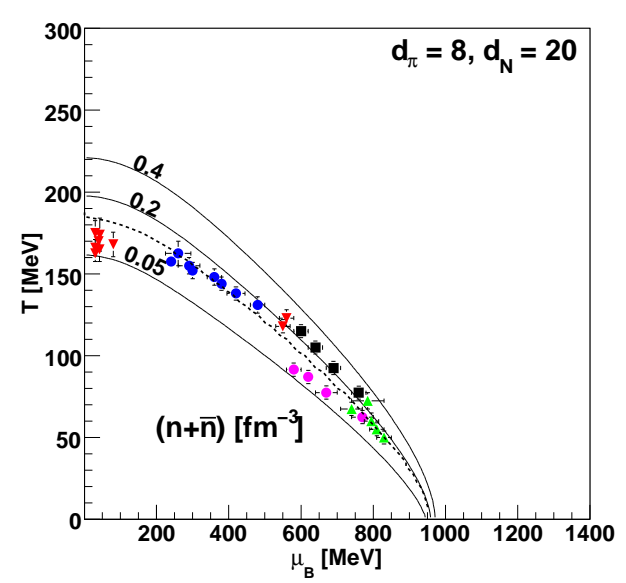

(b)

FIG. 3: Entropy $s$ divided by $T^{3}$ (a) and total baryon density $n+\bar{n}$ (b) as functions of temperature $T$ and the baryon chemical potential $\mu_{B}$ for the schematic resonance gas model with 8 pion and 20 nucleon degrees of freedom. The dashed curves represent the phenomenological lines of constant: $s\left(T^{f}, \mu_{B}^{f}\right) / T^{3}=7$ in (a) and $n_{B}\left(T^{f}, \mu_{B}^{f}\right)+\bar{n}_{B}\left(T^{f}, \mu_{B}^{f}\right)=0.12 \mathrm{fm}^{-3}$ in (b).

in the relaxation time for flavor equilibration. Already for a schematic resonance gas consisting of pions and nucleons with artificially enhanced numbers of degrees of freedom we could demonstrate that the kinetic freeze-out condition can provide quantitative agreement with the phenomenological freeze-out curve. The further development of this approach would clearly require the replacement of the schematic resonance gas model with the one based on the full spectrum of hadronic resonances and detailed studies of their influence on the chiral condensate. To this end generalized models for hadron resonance masses are required, which reveal their dependence on the current quark mass like, e.g., the model of Leupold [46]. However, it is very likely that with such extension the model results quantifying freeze-out conditions will still be consistent with phenomenological findings.

\section{Acknowledgements}

We acknowledge partial support by the Helmholtz International Center (HIC) for FAIR. The work of D.B. and K.R. is supported by the Polish Ministry for Science and Higher 
Education. K.R. received partial support of ExtreMe Matter Institute (EMMI).

[1] F. Karsch and K. Redlich, Phys. Lett. B 695, 136 (2011).

[2] P. Braun-Munzinger, K. Redlich and J. Stachel, in: Quark Gluon Plasma 3, Eds. R.C. Hwa and Xin-Nian Wang, World Scientific (2003), pp. 491-599; arXiv:nucl-th/0304013.

[3] P. Braun-Munzinger, D. Magestro, K. Redlich and J. Stachel, Phys. Lett. B 518, 41 (2001).

[4] J. Cleymans, K. Redlich, Phys. Rev. Lett. 81, 5284-5286 (1998).

[5] J. Cleymans, K. Redlich, Phys. Rev. C60, 054908 (1999).

[6] J. Cleymans, H. Oeschler, K. Redlich and S. Wheaton, Phys. Rev. C 73, 034905 (2006).

[7] J. Cleymans, H. Oeschler, K. Redlich and S. Wheaton, J. Phys. G 32, S165 (2006).

[8] F. Karsch and E. Laermann, Phys. Rev. D 50, 6954 (1994).

[9] L. McLerran and R. D. Pisarski, Nucl. Phys. A 796, 83 (2007).

[10] A. Andronic, D. Blaschke, P. Braun-Munzinger et al., Nucl. Phys. A837, 65-86 (2010).

[11] V. Magas and H. Satz, Eur. Phys. J. C 32, 115 (2003).

[12] V. D. Toneev, J. Cleymans, E. G. Nikonov et al., J. Phys. G G27, 827-832 (2001).

[13] P. Braun-Munzinger, J. Stachel and C. Wetterich, Phys. Lett. B 596, 61 (2004)

[14] B. J. Schaefer, J. M. Pawlowski and J. Wambach, Phys. Rev. D 76, 074023 (2007).

[15] D. Blaschke, M. Buballa, A. E. Radzhabov and M. K. Volkov, Yad. Fiz. 71, 2012 (2008).

[16] S. Roessner, T. Hell, C. Ratti and W. Weise, Nucl. Phys. A 814, 118 (2008).

[17] D. Blaschke, M. Buballa, A. E. Radzhabov and M. K. Volkov, Nucl. Phys. Proc. Suppl. 198, $51(2010)$.

[18] V. Skokov, B. Stokic, B. Friman and K. Redlich, Phys. Rev. C 82, 015206 (2010).

[19] V. Skokov, B. Friman and K. Redlich, arXiv:1008.4570 [hep-ph].

[20] A. E. Radzhabov, D. Blaschke, M. Buballa and M. K. Volkov, arXiv:1012.0664 [hep-ph].

[21] K. Fukushima, Prog. Theor. Phys. Suppl. 151 (2003) 171.

[22] K. Fukushima, Phys. Lett. B 591, 277 (2004).

[23] K. Fukushima, Phys. Rev. D 68, 045004 (2003).

[24] K. Fukushima, Phys. Lett. B 553, 38 (2003).

[25] C. Ratti, M. A. Thaler and W. Weise, Phys. Rev. D 73 (2006) 014019.

[26] S. Roessner, C. Ratti and W. Weise, Phys. Rev. D 75, 034007 (2007). 
[27] C. Sasaki, B. Friman and K. Redlich, Phys. Rev. D 75, 074013 (2007).

[28] J. Hüfner and B. Povh, Phys. Rev. D 46, 990 (1992).

[29] B. Povh, J. Hüfner, Phys. Lett. B 245, 653 (1990).

[30] K. Martins, D. Blaschke and E. Quack, Phys. Rev. C 51, 2723 (1995).

[31] H. J. Hippe and S. P. Klevansky, Phys. Rev. C 52, 2172 (1995).

[32] D. Blaschke and P. C. Tandy, in: Understanding Deconfinement in QCD, World Scientific (2000), pp. 218-230; arXiv:nucl-th/9905067.

[33] D. Blaschke, G. Burau, Yu. L. Kalinovsky, P. Maris and P. C. Tandy, Int. J. Mod. Phys. A 16, 2267 (2001).

[34] P. Zhuang, J. Hüfner and S. P. Klevansky, Nucl. Phys. A 576, 525 (1994).

[35] J. Hüfner, S. P. Klevansky, P. Zhuang and H. Voss, Annals Phys. 234, 225 (1994).

[36] H. Hansen, W. M. Alberico, A. Beraudo, A. Molinari, M. Nardi and C. Ratti, Phys. Rev. D 75, 065004 (2007).

[37] T. Hell, S. Roessner, M. Cristoforetti and W. Weise, Phys. Rev. D 79, 014022 (2009).

[38] See e.g., J. Berges, N. Tetradis and C. Wetterich, Phys. Rept. 363, 223 (2002).

[39] B. J. Schaefer, J. M. Pawlowski and J. Wambach, Phys. Rev. D 76, 074023 (2007);

B. Stokic, B. Friman and K. Redlich, Eur. Phys. J. C 67, 425 (2010);

E. Nakano, B. J. Schaefer, B. Stokic, B. Friman and K. Redlich, Phys. Lett. B 682, 401 (2010);

V. Skokov, B. Friman and K. Redlich, arXiv:1008.4570 [hep-ph];

T. K. Herbst, J. M. Pawlowski, B. -J. Schaefer, Phys. Lett. B 696, 58 (2011).

[40] T. D. Cohen, R. J. Furnstahl and D. K. Griegel, Phys. Rev. C 45, 1881 (1992).

[41] J. Gasser and H. Leutwyler, Phys. Lett. B 184, 83 (1987).

[42] M. Buballa, Phys. Rept. 407, 205 (2005).

[43] H. Grigorian, Phys. Part. Nucl. Lett. 4, 223 (2007).

[44] W. Ebeling, W.-D. Kraeft, D. Kremp, G. Röpke, Quantum Statistics of Charged Many-Particle Systems, Plenum, New York (1986).

[45] D. Blaschke, F. Reinholz, G. Ropke and D. Kremp, Phys. Lett. B 151, 439 (1985).

[46] S. Leupold, J. Phys. G 32, 2199-2218 (2006). 